\title{
Mecanismo explosivo de polinização em Periandra mediterranea (Vell.) Taub. (Fabaceae) na Reserva Biológica Guaribas, Paraíba, Brasil
}

\author{
Andressa Cavalcante Meireles ${ }^{1 *}$ \\ Joel Araújo Queiroz ${ }^{2}$ \\ Zelma Glebya Maciel Quirino $^{3}$
${ }^{1}$ Programa de Pós-Graduação em Ecologia de Ecótonos, Núcleo de Estudos Ambientais Universidade Federal do Tocantins, Rua Três, QD. $17 \mathrm{~s} / \mathrm{n}$ Caixa Postal 136, CEP 77500-000, Porto Nacional - TO, Brasil ${ }^{2}$ Programa de Pós-Graduação em Biologia Vegetal, Departamento de Botânica Centro de Ciências Biológicas, Universidade Federal de Pernambuco, Recife - PE, Brasil Universidade Federal da Paraíba, Rio Tinto - PB, Brasil
* Autor para correspondência
andressaecologia@gmail.com \\ ${ }^{3}$ Departamento de Engenharia e Meio Ambiente, Centro de Ciências Aplicadas e Educação
}

Submetido em 10/03/2015

Aceito para publicação em 21/09/2015

\section{Resumo}

A espécie Periandra mediterranea (Vell.) Taub. apresenta flores papilionáceas e bilabiadas o que proporciona um complexo mecanismo de polinização. A polinização e a reprodução de $P$. mediterranea foram estudadas no período de novembro/2009 a outubro/2011 na Reserva Biológica Guaribas, Paraíba, Brasil. As pétalas são modificadas em uma quilha, que protege os estames e o estigma; duas alas, que envolvem a quilha, e um estandarte, que serve de plataforma de pouso para o visitante floral. O mecanismo de polinização é o explosivo, no qual as abelhas Xylocopa frontalis, Acanthopus excellens e Epicharis sp. apresentavam o comportamento de pousar no estandarte e, auxiliadas pelo seu peso, eram capazes de expor os órgãos reprodutivos que se encontram inclusos no complexo alas-quilha. Como consequência, os órgãos reprodutivos da flor contatavam a região dorsal do corpo da abelha, onde o pólen ficava depositado, caracterizando a polinização nototríbica. Testes reprodutivos demonstraram frutificação de $20 \%$ em autopolinização espontânea, $33 \%$ em autopolinização manual, $33 \%$ em polinização cruzada manual e $100 \%$ no grupo controle, enquanto que em apomixia não houve sucesso reprodutivo. Os resultados demonstraram que a espécie é autocompatível, mas depende de polinizadores para garantir o seu sucesso reprodutivo.

Palavras-chave: Autocompatibilidade; Flores com quilha invertida; Melitofilia

\section{Abstract}

Explosive pollination mechanism in Periandra mediterranea (Vell.) Taub. (Fabaceae) in the Guaribas Biological Reserve, Paraíba, Brazil. Periandra mediterranea (Vell.) Taub. has papilionaceous flowers with a complex pollination mechanism. This study examined pollination and reproduction in $P$. mediterranea from 
November 2009 to October 2011 at the Guaribas Biological Reserve, Paraíba, Brazil. The petals are modified in a keel that protects the stamens and stigma; two wings surround the keel, and a standard that serves as landing platform for floral visitors. Periandra mediterranea exhibits an explosive type pollination mechanism in which the bee species Xylocopa frontalis, Acanthopus excellens and Epicharis sp., land on the standard and, due to body weight, expose the reproductive organs in the wing-keel complex. As a result, the reproductive organs of the flower come into contact with the dorsal region of the bee body, depositing pollen (i.e., nototríbic pollination). Reproductive assays showed $20 \%$ fruiting in spontaneous auto-pollination, $33 \%$ in manual autopollination, $33 \%$ in manual cross-pollination, and $100 \%$ in the control group, with no reproductive success while in apomixis. These results demonstrate self-compatibility in this species, however it depends on pollinators to ensure reproductive success.

Key words: Inverted keel flowers; Melittophily; Self-compatibility

\section{Introdução}

A subfamília Papilionoideae compreende cerca de 400 gêneros, sendo a maior entre as subfamílias das Fabaceas (JOLY, 1991). As espécies desta subfamília apresentam características florais distintas e bem elaboradas, que resultaram em uma diversidade de mecanismos de polinização (STIRTON, 1981). Dentro dessas características são destacadas as adaptações de suas flores para serem visitadas por insetos, como: perfume adocicado, antese diurna, cores vistosas e variada oferta de recursos (néctar, pólen) (ENDRESS, 1994). Esses atributos estão relacionados à melitofilia, síndrome de polinização por abelhas (FAEGRI; VAN DER PIJL, 1979).

Além dessas características, a maioria das Papilionoideae (aproximadamente 30 tribos) apresenta flores zigomórficas e pentâmeras (TUCKER, 2003), com pétalas diferenciadas em: duas alas; duas pétalas unidas que formam a quilha e uma pétala que forma o estandarte (ARROYO, 1981; TUCKER, 2003). Neste tipo floral, denominado flor bilabiada (sensu WESTERKAMP; CLABEN-BOCKHOFF, 2007), as partes florais (alas e quilhas) são ativamente manuseadas pelos visitantes durante a busca de recursos florais, sendo relacionadas com o comportamento e morfologia de espécies de abelhas (FAEGRI; VAN DER PIJL, 1979; ARROYO, 1981; TUCKER, 1987; 2003; WESTERKAMP; WEBER, 1999). Nessa subfamília a deposição e a remoção de pólen podem acontecer por intermédios de quatro mecanismos: valvular, bomba, escova e explosiva (ARROYO, 1981; YEO, 1993; WESTERKAMP, 1997;
WESTERKAMP; WEBER, 1999; GALLONI et al., 2007; LE ROUX; VAN WYK, 2012; ALEMÁN et al., 2014).

As flores de quilha invertida foram incluídas no grupo funcional de flores bilabiadas devido à sua organização ressupinada e transferência de pólen nototríbica, ou seja, na região dorsal do corpo dos visitantes florais (WESTERKAMP; CLABENBOCKHOFF, 2007; AMARAL-NETO et al., 2015). Flores de quilha invertida, que possuem o mecanismo explosivo de abertura e liberação de pólen, precisam que uma pressão seja aplicada na pétala estandarte, esta pressão é exercida pelo peso corporal em conjunto com os movimentos do visitante floral, que por sua vez utiliza esta pétala como plataforma de pouso, como consequência, as pétalas do complexo alasquilha se separam liberando os elementos reprodutivos repentinamente, lançando o pólen contra o polinizador (BAKER, 1970), dessa forma apenas alguns visitantes florais podem ativar eficientemente este mecanismo de polinização. Após a exposição dos órgãos reprodutivos o mecanismo explosivo é desativado podendo perder sua funcionalidade, e as pétalas não mais retornam a sua posição original (ARROYO, 1981; GALLONI; CRISTOFOLINI, 2003; GALLONI et al., 2007; ALEMÁN et al., 2014). No entanto, existem casos em que as pétalas podem retornar à sua posição original (GALLONI; CRISTOFOLINI, 2003; SOLOMON RAJU; PURNACHANDRA RAO, 2006).

A espécie Periandra mediterranea (Vell.) Taub., da tribo Phaseoleae, apresenta flores tipicamente 
papilionáceas (FUNCH; BARROSO, 1999). Esta espécie é nativa do Brasil, com distribuição em todas as regiões, sendo encontrada principalmente nos domínios fitogeográficos da Amazônia, Caatinga, Cerrado e Mata Atlântica (QUEIROZ, 2015).

Para o presente trabalho, selecionamos como objeto de estudo a espécie Periandra mediterranea, com o objetivo de investigar o mecanismo de polinização explosivo nessa planta. A espécie apresenta flores bilabiadas, com o estandarte assumindo o papel de estrutura de pouso e o complexo alas-quilha conferindo proteção às anteras (pólen) e ao estigma e tendo função importante no mecanismo de exposição dessas partes reprodutivas durante o contato com o visitante floral. Buscou-se responder as seguintes questões: (1) como ocorre o mecanismo explosivo de polinização em $P$. mediterranea? (2) quais visitantes florais são capazes de acionar eficientemente o mecanismo explosivo de polinização? (3) qual é o sistema de reprodução? e (4) há dependência de vetores de pólen para a formação de frutos?

\section{Material e Métodos}

\section{Área de estudo}

O trabalho foi realizado durante o período de novembro de 2009 a outubro de 2011 na Reserva Biológica Guaribas, localizada nos municípios de Rio Tinto e de Mamanguape (6 $6^{\circ} 0^{\prime} 53^{\prime}$ S e $35^{\circ} 09^{\prime} 59^{\prime} \mathrm{W}$ ), litoral norte da Paraíba. A reserva possui uma área superfície total de 4.321 ha, sendo dividida em três áreas. A área II foi o local escolhido como área do estudo e está localizada em Mamanguape, com 3.378 ha, onde está situada a sede da reserva, apresenta manchas de cerrado também conhecidos como tabuleiros paraibanos, entretanto, conta com uma cobertura vegetal predominante de Mata Atlântica.

\section{Espécie estudada}

Periandra mediterranea (Vell.) Taub. é um arbusto ereto ou escandente, podendo apresentar até $3,5 \mathrm{~m}$ de altura; indumento esparso a densamente pubérulo ou pubérulo e seríceo, ocasionalmente subvelutino, canescente, recobrindo as partes vegetativas, ou às vezes ausente; apresenta inflorescências terminais ou axilares, com pedúnculo variando de subséssil até 10 cm comprimento; papilionáceas, com ápice assimétrico (FUNCH; BARROSO, 1999).

Materiais botânicos da espécie estudada foram depositados no Herbário Lauro Pires Xavier/JPB da Universidade Federal da Paraíba, sob o número de registro 52.225.

\section{Morfologia floral}

Foram coletados flores e frutos para análise da morfometria e biologia floral. As medidas do comprimento da base para o ápice das pétalas (alas, quilha e estandarte) e dos órgãos reprodutivos (estames e pistilos) $(\mathrm{n}=30)$ foram realizadas com o auxilio de paquímetro digital. A razão pólen/óvulo (CRUDEN; HERMANN-PARKER, 1979) foi determinada através da contagem direta do número de grãos de pólen e de óvulos por flor $(n=20)$. A viabilidade polínica foi estimada pela contagem de grãos de pólen corados em solução de carmim acético 2\% (RADFORD et al., 1974). Para determinar a razão semente/óvulo dividiuse o número médio de sementes maduras encontradas nesses frutos $(\mathrm{n}=30)$ pelo número de óvulos por flor $(\mathrm{n}=30)$ (WIENS, 1984).

Estimou-se a quantidade média de flores produzidas por indivíduo $(\mathrm{n}=20)$ e o número médio de frutos por indivíduo $(\mathrm{n}=20)$ em duas subpopulações acompanhadas. A razão fruto/flor foi estimada pela divisão do número médio de flores/indivíduo pelo número médio de frutos/indivíduos $(\mathrm{n}=20)$.

\section{Antese}

A antese floral foi acompanhada em flores $(n=30)$ devidamente marcadas e enumeradas.

Para determinação da presença/ausência de néctar e pólen, botões florais em pré-antese $(n=30)$ foram encobertos com sacos de tule e monitorados durante toda duração da antese.

Para determinar o período de receptividade estigmática, foi utilizada solução de peróxido de 
hidrogênio (ZEISLER, 1938) no estigma de flores durante todo período de antese $(n=80)$.

\section{Mecanismo de polinização e visitantes florais}

Os animais visitantes foram observados diretamente no campo, em plantas focais $(\mathrm{n}=30)$ somando $375 \mathrm{~h}$ de observações diurnas. Foram registrados o horário e a duração das visitas, a frequência (número de visitas por intervalo de tempo) e o comportamento dos animais, ou seja, se a visita provocava exposição das estruturas reprodutivas das flores e contato de tais estruturas com a parte dorsal do corpo do visitante floral.

Sendo assim, os visitantes florais foram classificados em três categorias baseadas em critérios sugeridos por Inouye (1980): (1) Polinizador efetivo: quando a visita ativou o mecanismo de polinização explosiva, possibilitando contato da parte dorsal do corpo do visitante com as estruturas reprodutivas da flor, promovendo assim polinização efetiva; (2) Polinizador ocasional: quando a visita ativou o mecanismo de polinização explosiva, mas, devido ao posicionamento inadequado do animal na flor, não ocorria o contato das estruturas reprodutivas da flor com a parte dorsal do corpo do visitante, promovendo polinização apenas ocasionalmente; e (3) Pilhadores: quando a visita proporcionou a exploração dos recursos florais sem a ativação do mecanismo explosivo de polinização, dessa forma não promovendo contato entre o corpo do visitante e as estruturas reprodutivas da flor. Sendo também considerados pilhadores os animais que apresentavam o comportamento de explorar o néctar realizando perfurações na corola das flores, sem acionar o mecanismo explosivo.

\section{Experimentos de polinização}

A análise do sistema reprodutivo foi realizada através de testes de polinização em flores de diferentes indivíduos ( $\mathrm{n}=10$ indivíduos) de $P$. mediterranea, sendo realizados os seguintes tratamentos: polinização cruzada manual $(n=30)$ (flores no período de pré-antese foram emasculadas e a polinização foi feita esfregando-se sobre os estigmas receptores as anteras de uma flor recém aberta de outro indivíduo), autopolinização manual $(n=30)$ (o estigma da flor em período de pré-antese recebeu o pólen das anteras da própria flor com auxílio de um pincel), autopolinização espontânea $(n=30)$ (botões florais foram isoladas até a possível formação do fruto) e apomixia $(n=30)$ (as anteras foram eliminadas em flores no período de pré-antese). Para quantificar a polinização natural (controle), flores $(n=30)$ visitadas pelo polinizador efetivo (INOUYE, 1980; FREITAS, 2013), foram marcadas e acompanhadas quanto à formação ou não de frutos. As flores de todos os tratamentos foram ensacadas com tule para evitar contato com possíveis visitantes florais e acompanhadas até a formação ou não dos frutos.

\section{Resultados}

\section{Morfologia floral}

Periandra mediteranea apresenta inflorescências cimosas com flores tipicamente papilionáceas com cerca de 2-3 botões por inflorescência sendo que 1-2 se encontram abertos a cada dia e 1-2 inflorescência por ramo. As pétalas são de colorações violáceas e modificadas em quilha $(22,99 \pm 0,43 \mathrm{~mm}$ comprimento) que envolve e protege os órgãos reprodutivos estames e estigma; alas (24,11 $\pm 0,13 \mathrm{~mm}$ comprimento) que envolvem a quilha e a estandarte $(32,51 \pm 0,74 \mathrm{~mm}$ comprimento) que serve de plataforma de pouso para o visitante floral (Figura 1).

FIGURA 1: Vista frontal da flor caracteristicamente melitófila de Periandra mediterranea (Vell.) Taub. (Fabaceae), destacando-se as pétalas modificadas: $\mathrm{a}-$ Alas; $\mathrm{b}-$ Quilha; c - Estandarte; seta - guia de néctar.

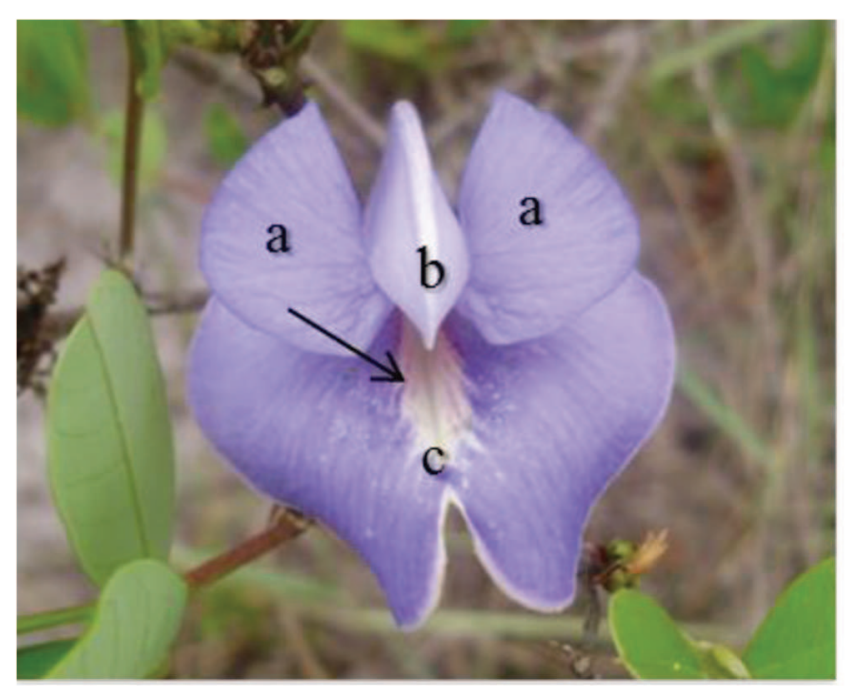


Os recursos florais consistem em pólen e néctar (ENDRESS, 1994). O nectário nesta espécie encontrase entre a base da coluna estaminal e do ovário, sendo esse último súpero, o pistilo e estames apresentaram tamanhos de $30 \pm 0,63$ e $26 \pm 0,51 \mathrm{~mm}$ respectivamente, o que proporcionaram hercogamia, por conseguinte, durante a visita os polinizadores tocavam primeiro o estigma e depois as anteras. O androceu é diplostêmone, gamostêmone e diadelfo.

A razão pólen/ovulo foi de aproximadamente $2220 / 8=277,5$ indicando a espécie como xenogama facultativa. A viabilidade polínica foi de $98 \%$. A razão entre o número de sementes e o número de óvulos foi de $6 / 8=0,75$. A razão entre o número de flores e a produção de frutos por indivíduo foi de 5/3=1,66.

\section{Antese}

$\mathrm{Na}$ fase de pré-antese, o estandarte encobre as alas, a quilha e os órgãos reprodutivos. A antese é diurna e sincrônica tendo início às $04 \mathrm{~h} 00 \mathrm{~min}$ sendo que as $05 \mathrm{~h} 00 \mathrm{~min}$ todas as flores se encontravam abertas. A antese inicia com a distensão lenta dos lobos da corola, ocasião em que as anteras iniciam a deiscência, permanecendo inclusas na quilha sendo expostas apenas após a visita do polinizador efetivo. O estigma encontra-se receptivo apenas durante o primeiro dia de antese entre às $04 \mathrm{~h} 00 \mathrm{~min}$ e $16 \mathrm{~h} 00 \mathrm{~min}$, ocasião em que os grãos de pólen estão disponíveis, existindo também a presença de néctar e perfume. A partir das 04h00min do segundo dia as flores começam a fechar, a duração da antese é, portanto, de um dia ou 24 h. Logo após ocorre um processo de mudança da corola, que se torna completamente murcha e de coloração amarronzada após 48 horas do início do processo da antese, em seguida as pétalas começam a cair, sendo possível observar a inicial formação do fruto.

\section{Mecanismo de polinização e visitantes florais}

Muitas espécies visitaram as flores de Periandra mediterranea (Tabela 1) entre elas se destacaram as abelhas Xylocopa frontalis, Apis mellifera, Epicharis sp. e Acanthopus excellens. Foram registrados também o beija-flor Phaethornis pretrei, as vespas Polybia sp. 1 e Polybia sp. 2 e ainda o esfingídeo Urbanus simplicius (Figura 2).

TABELA 1: Visitantes florais de Periandra mediterranea (Vell.) Taub. na Reserva Biológica Guaribas, Paraíba, Brasil. $\mathrm{F}=$ frequente $(>35 \%), \mathrm{P}=$ pouco frequente $(16-36 \%), \mathrm{R}=$ raro $(6-15 \%), \mathrm{M}=$ muito raro $(<5 \%) ; \mathrm{N}=$ néctar; $\mathrm{Po}=$ pólen $; \mathrm{Pe}=$ polinizador efetivo, $\mathrm{Pi}=$ pilhador; $\mathrm{Poc}=$ Polinizador ocasional .

\begin{tabular}{lcccccc}
\hline \multicolumn{1}{c}{ Visitantes florais } & $\begin{array}{c}\text { Total de } \\
\text { visitas } \\
(\mathbf{n})\end{array}$ & $\begin{array}{c}\text { Total de } \\
\text { visitas } \\
(\mathbf{\%})\end{array}$ & Frequência & $\begin{array}{c}\text { Recurso } \\
\text { coletado }\end{array}$ & $\begin{array}{c}\text { Duração } \\
\text { das visitas } \\
\text { (segundos) }\end{array}$ & $\begin{array}{c}\text { Eficiência da } \\
\text { polinização }\end{array}$ \\
\hline Xylocopa frontalis (Olivier, 1789) & 79 & 60,76 & $\mathrm{~F}$ & $\mathrm{~N} / \mathrm{Po}$ & 08 & $\mathrm{Pe}$ \\
Acanthopus excellens (Schrottky, 1902) & 13 & 10,1 & $\mathrm{R}$ & $\mathrm{N} / \mathrm{Po}$ & 10 & $\mathrm{Poc} / \mathrm{Pi}$ \\
Apis melifera (Linnaeus, 1758) & 05 & 3,84 & $\mathrm{M}$ & $\mathrm{N}$ & 06 & $\mathrm{Pi}$ \\
Epicharis sp. & 05 & 3,84 & $\mathrm{M}$ & $\mathrm{N} / \mathrm{Po}$ & 08 & $\mathrm{Poc} / \mathrm{Pi}$ \\
Polybia sp. 1 & 15 & 11,53 & $\mathrm{M}$ & $\mathrm{N}$ & 150 & $\mathrm{Pi}$ \\
Polybia sp. 2 & 03 & 2,31 & $\mathrm{M}$ & $\mathrm{N}$ & 160 & $\mathrm{Pi}$ \\
Urbanus simplicius (Stoll, 1790) & 03 & 2,31 & $\mathrm{M}$ & $\mathrm{N}$ & 120 & $\mathrm{Pi}$ \\
Phaethornis pretrei (Lesson \& Delattre, 1839) & 07 & 5,38 & $\mathrm{R}$ & $\mathrm{N}$ & 06 & $\mathrm{Pi}$ \\
\hline
\end{tabular}


FIGURA 2: Principais visitantes florais de Periandra mediterranea (Vell.) Taub. (Fabaceae) na Reserva Biológica Guaribas, Paraíba, Brasil: a) Xylocopa frontalis (polinizador efetivo, seta: deposição nototríbica de pólen); b) Acanthopus excellens (polinizador ocasional e pilhador, seta: exposição lateral dos órgãos reprodutivos da planta); c) Epicharis sp. (polinizador ocasional e pilhador); d) Urbanus simplicius (pilhador); e) Polybia sp. 1 (pilhador); e) Polybia sp. 2 (pilhador).

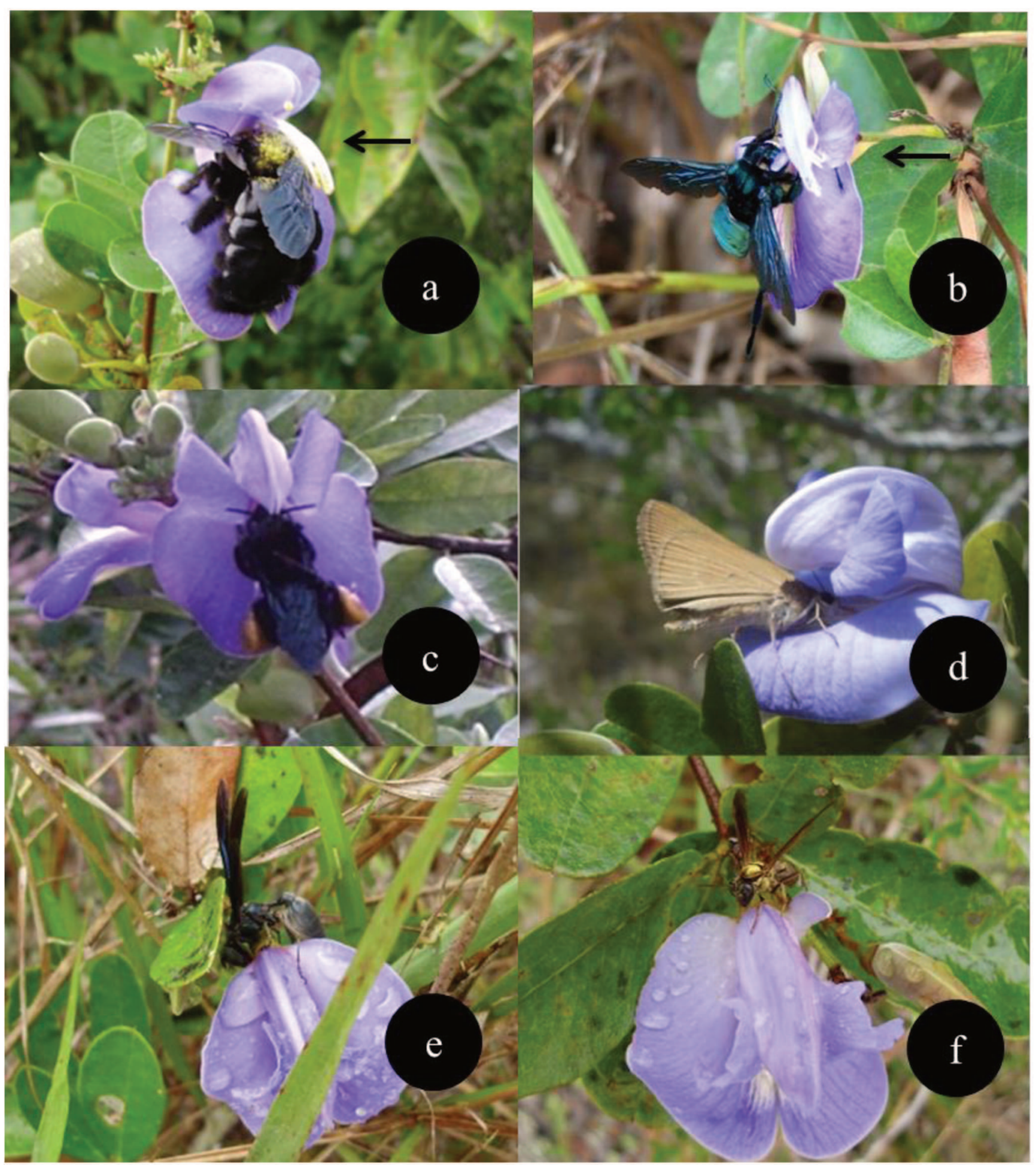


As visitas de $X$. frontalis ocorreram principalmente nos intervalos entre $07 \mathrm{~h} 00 \mathrm{~min}$ e $08 \mathrm{~h} 00 \mathrm{~min}$ período de alta receptividade estigmática e disponibilidade polínica. As abelhas A. excellens, A. mellifera, Epicharis sp. e as vespas Polybia sp. 1 e Polybia sp. 2 foram registrados em todo período da manhã. $\mathrm{O}$ beija-flor $P$. pretrei foi observado realizando visitas nas flores de $P$. mediterranea principalmente no intervalo de entre as $07 \mathrm{~h} 00 \mathrm{~min}$ e $08 \mathrm{~h} 00 \mathrm{~min}$, sendo ainda realizado o seu registro entre $15 \mathrm{~h} 00 \mathrm{~min}$ e $16 \mathrm{~h} 00 \mathrm{~min}$. O esfingídeo U. simplicius foi observado apenas no final da tarde (Figura 3).

A Xylocopa frontalis é o polinizador efetivo e principal de $P$. mediterranea, realizando um elevado número de visitas ás flores de modo adequado e eficiente, coletando pólen de forma passiva e néctar. O mecanismo de polinização é o explosivo, no qual a abelha apresentava o comportamento de pousar no estandarte e, auxiliado pelo peso exercido com seu corpo juntamente com seus movimentos corporais vibratórios, é capaz de expor os órgãos reprodutivos que se encontram inclusos no complexo alas-quilha, posteriormente, introduziam o aparelho bucal na base dos elementos florais para alcançar o néctar. Como consequência, os órgãos reprodutivos da flor eram liberados e contatavam a região dorsal do corpo da abelha, onde o pólen ficava depositado, caracterizando a polinização nototríbica (Figura 2a).

Após a polinização efetiva, o mecanismo explosivo de polinização era desativado e as pétalas do complexo alas-quilha não mais retornavam a sua posição original (Figura 4).

A abelha Acanthopus excellens foi considerada como polinizador ocasional devido ao seu comportamento, realizando tanto visitas legítimas coletando pólen de forma passiva e o néctar, como também, visitas ilegítimas onde realizava apenas a pilhagem de néctar sem acionar o mecanismo explosivo de exposição dos órgãos reprodutivos da planta, além disso, foi observado que esse animal pode expor os órgãos reprodutivos da flor lateralmente sem tocá-los (Figura 2b). Quando comparado ao polinizador efetivo da espécie $(X$. frontalis), A. excellens realizou uma quantidade menor de visitas às flores.

FIGURA 3: Proporção de visitas por intervalos de horários ás flores de Periandra mediterranea (Vell.) Taub. (Fabaceae) na Reserva Biológica Guaribas, Paraíba, Brasil.

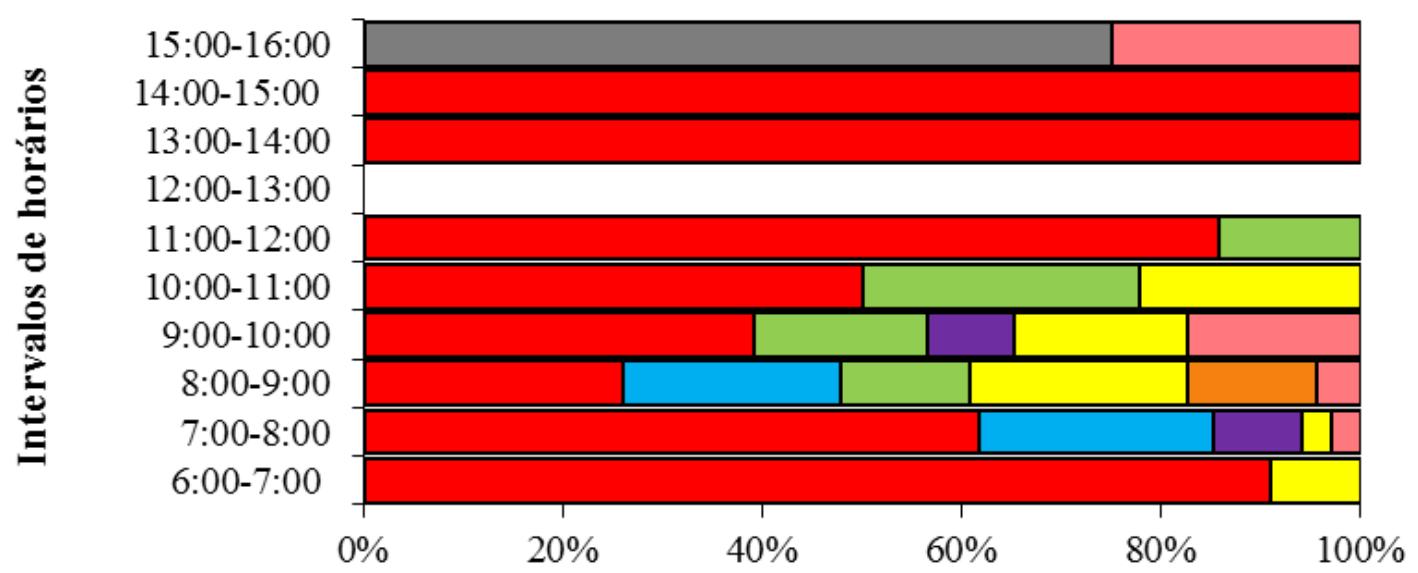

Frequência de visitas

$\square$ Xylocopa frontalis

$\square$ Polybia sp. 1 $\square$ Acanthopus excellens

$\square$ Polybia sp. 2
Apis melifera

Urbanus simplicius
Epicharis sp.

$\square$ Phaethornis pretrei 
FIGURA 4: Vista lateral da flor de Periandra mediterranea (Vell.) Taub. (Fabaceae), (a) antes e (b) após ativação do mecanismo explosivo de polinização pela abelha Xylocopa frontalis; seta - órgãos reprodutivos expostos.

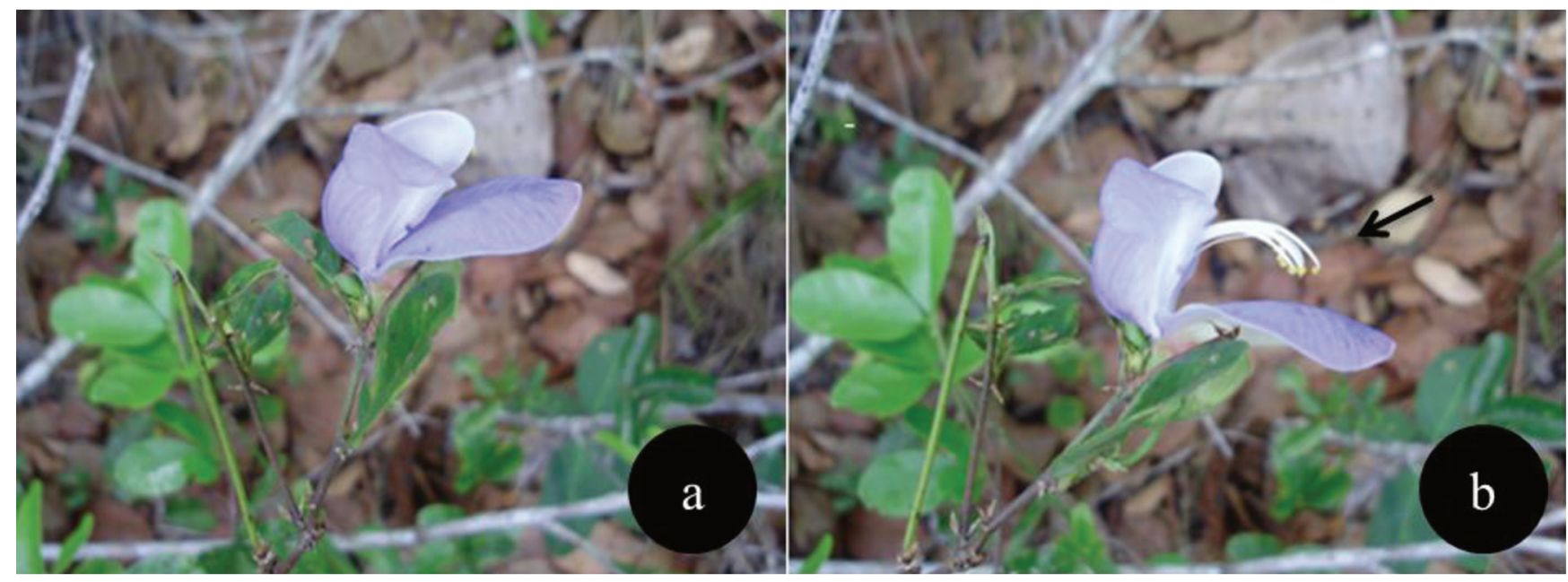

A abelha Epicharis sp. foi considerada como polinizador ocasional, pois durante as visitas realizava tanto a polinização efetiva tocando os órgãos reprodutivos da planta e realizando a retirada de néctar e a coleta passiva de pólen, como também, podiam realizar apenas a pilhagem de néctar sem ativar o mecanismo explosivo.

A abelha Apis mellifera, as vespas Polybia sp. 1 e Polybia sp. 2 e o esfingídeo Urbanus simplicius são apenas pilhadores de néctar em $P$. mediterranea apresentando o comportamento de pousar sobre a estandarte e introduzir o aparelho bucal diretamente na base da corola, acessando o nectário sem ativar o mecanismo explosivo de polinização. Foi constatado ainda que as espécies de Polybia ssp. realizavam perfurações na corola da planta como forma de acessar o nectário floral.

O beija-flor Phaethornis pretrei é apenas um pilhador de néctar. Durante as visitas esta espécie introduzia o bico nas flores fazendo a retirada do néctar sem constatar nos órgãos reprodutivos da planta.

\section{Experimentos de polinização}

Testes de polinização demonstraram diferentes resultados na formação de frutos: na polinização cruzada manual o sucesso reprodutivo foi de $33 \%$; na autopolinização manual $33 \%$; na autopolinização espontânea natural 20\%; na polinização controle indicou $100 \%$ de sucesso na produção de frutos; na apomixia não ocorreu produção de frutos (Tabela 2). Tais resultados indicam que $P$. mediterranea é autocompatível.

TABELA 2: Características do sistema reprodutivo de Periandra mediterranea (Vell.) Taub. (Fabaceae) na Reserva Biológica Guaribas, Paraíba, Brasil.

\begin{tabular}{lccc}
\hline \multicolumn{1}{c}{ Tratamentos } & Flores & Frutos & $\begin{array}{c}\text { Sucesso } \\
(\%)\end{array}$ \\
\hline Apomixia & 30 & 0 & 0 \\
Autopolinização espontânea & 30 & 6 & 20 \\
Autopolinização manual & 30 & 10 & 33 \\
Polinização cruzada manual & 30 & 10 & 33 \\
Controle & 30 & 30 & 100 \\
\hline
\end{tabular}

\section{Discussão}

As observações realizadas no campo sobre o comportamento dos visitantes florais confirmaram que o arranjo morfológico das pétalas desempenha uma importante função na ativação do mecanismo explosivo, como foi sugerido para flores bilabiadas (STIRTON, 1981; WESTERKAMP, 1997; WESTERKAMP; WEBER, 1999; ETCHEVERRY et al., 2008; LE ROUX; VAN WYK, 2012; ALEMÁN et al., 2014; AMARALNETO et al., 2015). 
As flores de Periandra mediterranea apresentam diversos atributos morfológicos considerados por Endress (1994) e Faegri e van der Pijl (1979) como associados à melitofilia como forma tridimensional, zigomorfia, coloração violácea, presença de néctar, perfume adocicado e antese diurna. Essas características proporcionam um padrão de polinização capaz de restringir a diversidade de polinizadores que, além da necessidade de posicionar-se corretamente entre as peças florais, devem possuir o tamanho e peso adequados para exercer pressão suficiente para expor os órgãos reprodutivos (CÓRDOBA; COCUCCI, 2011).

Além disso, P. mediterranea apresenta um sistema de polinização compartilhado por outras Fabaceae da subfamília Faboideae, no qual os verticilos reprodutores são encobertos e protegidos pela quilha, e sua exposição acontece somente após a visita do polinizador. Essa tendência foi registrada em Centrosema pubescens Benth. (BORGES, 2006), Canavalia brasiliensis Mart. ex Benth. (GUEDES et al., 2009), Abrus precatorius L. e Abrus Pulchellus Wall. ex Thw. (AGBAGWA; OBUTE, 2007) e Sophora tomentosa L. (BRITO et al., 2010).

A transferência de pólen pelos polinizadores realizada através da deposição nototríbica é comum em plantas com flores bilabiadas (WESTERKAMP; CLABEN-BOCKHOFF, 2007; AMARAL-NETO et al., 2015), isso acontece devido a inversão da quilha, de forma que o estandarte assume o papel de estrutura de pouso, ao invés do complexo alas-quilha, que se encontra em posição invertida (ARROYO, 1981), essa foi provavelmente uma evolução de suas adaptações para o ajustamento de seus mecanismos de polinização para evitar perda de pólen e dessa forma aumentar a eficiência da polinização cruzada (AMARAL-NETO et al., 2015).

Logo após da ativação do mecanismo de polinização explosiva de $P$. mediterranea, foi constatado que os órgãos reprodutivos das flores não mais retornam a posição natural (Figura 4) assim como verificado em outros estudos (ver: ARROYO, 1981; SUZUKI, 2003; GALLONI et al., 2007) e, não foram registradas outras visitas pelo polinizador efetivo, o que indica que a liberação total dos grãos de pólen e o processo de polinização são ambos efetuados na primeira visita, além disso, a exposição dos órgãos pode agir como uma sinalização para $X$. frontalis que estas flores já foram visitadas e apresentam poucos recursos (pólen e néctar) para ofertar, estas informações também foram registradas para Desmodium incanum DC., D. subsericeum Malme e D. uncinatum (Jacq.) DC. (ALEMÁN et al., 2014).

O mecanismo de hercogamia observado na espécie estudada favorece a polinização cruzada realizada pelas abelhas, pois durante a visita a região dorsal dos animais toca primeiramente o estigma e depois as anteras, otimizando o aumento do fluxo polínico entre as populações (ver: ETCHEVERRY et al., 2010).

De acordo com Freitas (2013), a efetividade dos polinizadores é dada através da sua contribuição total para sucesso reprodutivo de uma determinada espécie de planta, portanto, isto pode ser refletido através da eficácia do polinizador e intensidade de visitação. A abelha $X$. frontalis foi considerada o único polinizador efetivo da espécie estudada, devido principalmente a grande eficácia de polinização e as altas taxas de visitação quando comparada aos demais polinizadores (Tabela 1).

A fauna associada de $P$. mediterranea foi semelhante a de Vigna caracalla (L.) Verdc. (ETCHEVERRY; TRUCCO ALEMÁN, 2008) sendo também caracterizada por abelhas do gênero Xylocopa que realizavam visitas legítimas enquanto a $A$. mellifera e esfingídeos restringiam a pilhar néctar.

Embora a síndrome de melitofilia seja predominante em Papilionoideae (ARROYO, 1981) espécies polinizadas por abelhas também podem ser polinizadas por aves e particularmente por beija-flores de modo legítimo apresentando um sistema de polinização generalista (ETCHEVERRY; TRUCCO ALEMÁN, 2005). Apesar de $P$. mediterranea apresentar morfologia floral papilionácea, o beija-flor Phaethornis pretrei registrado no estudo restringiu-se a pilhar o néctar.

Nos tratamentos de autopolinização espontânea, autopolinização manual, polinização cruzada e apomixia o baixo número de frutos formados é esperado para família Fabaceae devido ao alto índice de frutos abortados como já registrado em Cliricidia sepium (Jacq.) Steud. (KILL; DRUMOND, 2001), Senna sylvestris (Vell.) H. S. Irwin \& Barneby (CARVALHO; OLIVEIRA, 2003), Acacia mearnsii de Wild. (ALVES; MARINS- 
GORDER, 2009), Caesalpinia pyramidalis Tul. (LEITE; MACHADO, 2009), Senna multijuga (Rich.) H. S. Irwin \& Barneby (WOLOWSKI; FREITAS, 2010) Desmodium incanum, D. subsericeum e D. uncinatum (ALEMÁN et al., 2014). Apesar de P. mediterranea ser uma espécie autocompatível, o baixo sucesso nos testes reprodutivos demonstrou a importância dos polinizadores para garantir uma maior produção de frutos.

Diante do exposto conclui-se que $P$. mediterranea é dependente da ação do polinizador $X$. frontalis o que suporta a ideia que a ativação do mecanismo explosivo realizado por este animal é essencial para assegurar o sucesso reprodutivo da espécie, demonstrando que a manutenção desta abelha na área de estudo é fundamental para garantir o fluxo de pólen entre indivíduos de populações distintas e até mesmo distantes, contribuindo dessa forma, na produção de frutos por polinização cruzada.

\section{Agradecimentos}

Ao Conselho Nacional de Desenvolvimento Científico e Tecnológico (CNPq) pela bolsa de concedida a primeira autora. A todos que compõem a Reserva Biológica Guaribas pelo apoio no campo. Ao Laboratório de Ecologia Vegetal da Universidade Federal da Paraíba.

\section{Referências}

AGBAGWA, O. I.; OBUTE, G. C. Breeding system and pollination ecology of two Abrus species (Fabaceae) from tropical West Africa. Acta Botanica Croatica, Zagreb, v. 66, n. 2, p. 205-216, 2007.

ALEMÁN, M.; FIGUEROA-FLEMING, T.; ETCHEVERRY, A. SÜHRING, S. ORTEGA-BAES, P. The explosive pollination mechanism in Papilionoideae (Leguminosae): an analysis with three Desmodium species. Plant Systematics Evolution, Jena, v. 300, n. 1, p. 177-186, 2014.

ALVES, E. M. S.; MARINS-CORDER, M. P. Biologia reprodutiva de Acacia mearnsii De Wild. (Fabaceae) IV: visitantes florais. Revista Árvore, Viçosa, v. 33, n. 3, p. 443-450, 2009.

AMARAL-NETO, L.; WESTERKAMP, C.; MELO, G. A. R. From keel to inverted keel flowers: functional morphology of "upside down" papilionoid flowers and the behavior of their bee visitors. Plant Systematics Evolution, Jena, v. 301, p. n/a, 2015.

ARROYO, M. T. K. Breeding systems and pollination biology in Leguminosae. In: POLHILL, R. M.; RAVEN, P. H. (Ed.). Advances in legume systematics. Kew: Royal Botanic Gardens, 1981. p. 723-769.
BAKER, H. G. Two cases of bat pollination in Central America. Revista de Biologia Tropical, San José, v. 17, n. 2, p. 178-197, 1970.

BORGES, H. B. N. Biologia reprodutiva de Centrosema pudesncens Benth. (Fabaceae). Boletim Museu Paraense Emílio Goeldi, Ciências Naturais, Belém, v. 1, n. 1, p. 31-38, 2006.

BRITO, P. V. L. G.; PINHEIRO, M.; SAZIMA, M. Sophora tomentosa e Crotalaria vitellina (Fabaceae): biologia reprodutiva e interações com abelhas na restinga de Ubatuba, São Paulo. Biota Neotropica, Campinas, v. 10, n. 1, p. 185-192, 2010.

CARVALHO, D. A.; OLIVEIRA, P. E. Biologia reprodutiva e polinização de Senna sylvestris (Vell.) H.S. Irwin \& Barneby (Fabaceae, Caesalpinioideae). Revista Brasileira de Botânica, São Paulo, v. 26, n. 3, p. 319-328, 2003.

CÓRDOBA, A. S.; COCUCCI, A. A. Flower power: its association with bee power and floral functional morphology in Papilionate Legumes. Annals of Botany, Oxford, v. 108, p. 919-931, 2011.

CRUDEN, R. W.; HERMANN-PARKER, S. M. Butterfly pollination of Caesalpinia pulcherrima, with observations on a psychophilous syndrome. Journal of Ecology, Malden, v. 67, n. 1, p. $155-168,1979$.

ENDRESS, P. K. Diversity and evolutionary biology of tropical flower. Cambridge: Cabridge University Press, 1994. 420 p.

ETCHEVERRY, A. V.; ALEMAN, M. M.; FIGUEROA FLEMING, T; GOMEZ, C. A.; LOPEZ SPAHR, D. Anther-stigma separation in Desmodium species (Papilionoideae-Fabaceae) from Northwestern Argentina. Acta Horticulturae, Leuven, v. 918, p. 183-188, 2010.

ETCHEVERRY, A. V.; TRUCCO ALEMÁN, C. E. Reproductive biology of Erythrina falcata (Fabaceae: Papilionoideae). Biotropica, Malden, v. 37, n. 1, p. 54-63, 2005.

ETCHEVERRY, A. V.; TRUCCO ALEMÁN, C. E. Flower morphology, pollination biology and mating system of the complex flower of Vigna caracalla (Fabaceae: Papipionoideae). Annals of Botany, Oxford, v. 102, n. 3, p. 305-316, 2008.

FAEGRI, K.; VAN DER PIJL, L. The principles of pollination ecology. 3. ed. Oxford: Pergamon Press, 1979. 244 p.

FREITAS, L. Concepts of pollinator performance: is a simple aproach necessary to achieve a standardized terminology? Brazilian Journal of Botany, São Paulo, v. 36, n. 1, p. 3-8, 2013.

FUNCH, L. S.; BARROSO, G. M. Revisão taxonômica do gênero Periandra Mart. ex Benth. (Leguminosae, Papilionoideae, Phaseoleae). Revista Brasileira Botânica, São Paulo, v. 22 n. 3, p. 339-356, 1999.

GALLONI, M.; CRISTOFOLINI, G. Floral rewards and pollination in Cytiseae (Fabaceae). Plant Systematics Evolution, Jena, v. 238, p. 127-137, 2003.

GALLONI, M.; PODDA, L.; VIVARELLI, D.; CRISTOFOLINI, G. Pollen presentation, pollen-ovule ratios, and other reproductive traits in Mediterranean legumes (Fam. Fabaceae-Subfam. Faboideae). Plant Systematics Evolution, Jena, v. 266, n. 1, p. 147-164, 2007.

GUEDES, R. S.; QURINO, Z. G. M.; GONÇALVES, E. P. Fenologia reprodutiva e biologia da polinização de Canavalia brasiliensis Mart. ex Benth (Fabaceae). Biotemas, Florianópolis, v. 22, n. 1, p. 27-37. 2009. 
INOUYE, D. W. The terminology of floral larceny. Ecology, New York, v. 61, p. 1251-1253, 1980.

JOLY, A. B. Botânica: introdução à taxonomia vegetal. 10. ed. São Paulo: Companhia Editora Nacional, 1991. 462 p.

KILL, L. H. P.; DRUMOND, M. A. Biologia floral e sistema reprodutivo de Gliricidia sepium (Jacq.) Steud. (FabaceaePapilionoidae) na Região de Petrolina, Pernambuco. Ciência Rural, Santa Maria, v. 31, n. 4, p. 597-601, 2001.

LE ROUX, M. M.; VAN WYK, B. E. The systematic value of flower structure in Crotalaria and related genera of the tribe Crotalarieae (Fabaceae). Flora, Jena, v. 207, p. 414-426, 2012.

LEITE, A. V.; MACHADO, I. C. Biologia reprodutiva da "catingueira" (Caesalpinia pyramidalis Tul., LeguminosaeCaesalpinioideae), uma espécie endêmica da Caatinga. Revista Brasileira de Botânica, São Paulo, v. 32, n. 1, p. 79-88, 2009.

QUEIROZ, L. P. Periandra. In: FORZZA, R. C.; LEITMAN, P. M.; COSTA, A. F.; CARVAlHO JR., A. A.; PEIXOTO, A. L.; WALTER, B. M. T.; BICUDO, C.; ZAPPI, D.; COSTA, D. P.; LLERAS, E.; MARTINELLI, G.; LIMA, H. C.; PRADO, J.; STEHMANN, J. R.; BAUMGRATZ, J. F. A.; PIRANI, J. R.; SYLVESTRE, L.; MAIA, L. C.; LOHMANN, L. G.; QUEIROZ, L. P.; SILVEIRA, M.; COELHO, M. N.; MAMEDE, M. C.; BASTOS, M. N. C.; MORIM, M. P.; BARBOSA, M. R.; MENEZES, M.; HOPKINS, M.; SECCO, R.; CAVALCANTI, T. B.; SOUZA, V. C. (Ed.). Lista de espécies da flora do Brasil. 2015. Rio de Janeiro: Jardim Botânico do Rio de Janeiro. Disponível em: $<$ http:// floradobrasil.jbrj.gov.br/jabot/floradobrasil/FB29812>. Acesso em: 19 jun. 2015.

RADFORD, A. E.; DICKINSON, W. C.; MASSEY, J. R.; BELL, C. R. Vascular plant systematic. New York: Harper \& How Publishers, 1974. 891 p.

SOLOMON RAJU, A. J.; PURNACHANDRA RAO, S. Explosive pollen release and pollination as a function of nectar-feeding activity of certain bees in the biodiesel plant, Pongamia pinnata (L.) Pierre (Fabaceae). Current Science, Bangalore, v. 90, p. 7-10, 2006.
STIRTON, C. H. Petal sculpturing in Papilionoid legumes. In: POLHILL, R. M.; RAVEN, R. H. (Ed.). Advances in legume systematics. Kew: Royal Botanical Gardens, 1981. p. 771-788.

SUZUKI, N. Significance of flower exploding pollination on the reproduction of the Scotch broom, Cytisus scoparius (Leguminosae). Ecological Research, Tokyo, v. 18, n. 5, p. 523$532,2003$.

TUCKER, S. C. Floral initiation and development in legumes. In: STIRTON, C. H. (Ed.). Advances in legume systematics. Vol. 3. Kew: Royal Botanic Gardens, 1987. p. 183-239.

TUCKER, S. C. Floral development in legumes. Plant Physiology, Rockville, v. 131, n. 3, p. 911-926, 2003.

WESTERKAMP, C. Keel blossoms: bee flowers with adaptations against bees. Flora, Jena, v. 192, n. 1, p. 125-132, 1997.

WESTERKAMP, C.; CLAßEN-BOCKHOFF, R. Bilabiate flowers: the ultimate response to bees? Annals of Botany, Oxford, v. 100, p. 361-374, 2007.

WESTERKAMP, C.; WEBER, A. Keel flowers of the Polygalaceae and Fabaceae: a functional comparison. Botanical Journal of the Linnean Society, London, v. 129, n. 3, p. 207-221, 1999.

WIENS, D. Ovule survivorship, brood size, life story, breeding systems and reproductive success in plantes. Oecologia, New York, v. 64, n. 1, p. 47-53, 1984.

WOLOWSKI, M.; FREITAS, L. Sistema reprodutivo e polinização de Senna multijuga (Fabaceae) em Mata Atlântica Montana. Rodriguésia, Rio de Janeiro, v. 61, n. 2, p. 167-179, 2010.

YEO, P. F. Secondary pollen presentation: form, function and evolution. Plant Systematics and Evolution, Supplementum 6. Wien, New York: Springer-Verlag, 1993. 268 p.

ZEISLER, M. Uber die adgrenzung der eigentlichen narbenfleche mit hilfe von reaktionen. Beth. Botanisches Centralblatt, Jena, v. 58, n. 1, p. 308-318, 1938. 